Saudi Journal of Medicine

Abbreviated Key Title: Saudi J Med ISSN 2518-3389 (Print) |ISSN 2518-3397 (Online) Scholars Middle East Publishers, Dubai, United Arab Emirates Journal homepage: https://saudijournals.com/sjm

Original Research Article

\title{
Antimicrobial Therapy for Extended Spectrum Beta Lactamase Producing Uropathogenic Escherichia coli. Is Fosfomycin A Reasonable Alternative to Nitrofurantoin?
}

\author{
Dr. Nazia Khan ${ }^{1}$, Dr. Pragyan Swagatika Panda ${ }^{2 *}$, Dr. (Dir. Prof.) Man Mohan Mehndiratta ${ }^{3}$, Dr. Swati Sharma ${ }^{4}$, Dr \\ Megha Rastogi ${ }^{5}$, Dr. Shefali Gupta ${ }^{6}$, Dr. Neha Rana ${ }^{7}$ \\ ${ }^{1}$ Senior Resident, Department of Microbiology Maulana Azad Medical College and Lok Nayak hospital, New Delhi, India
${ }^{2}$ Assistant Professor, Department of Microbiology Janakpuri Super Speciality Hospital society, New Delhi, India
${ }^{3}$ Director JSSHS and HOD Neurology Janakpuri Super Speciality Hospital society, New Delhi, India
${ }^{4,5}$ Senior Resident, Department of Microbiology Janakpuri Super Speciality Hospital society, New Delhi, India
${ }^{6}$ Assistant Professor, Dept of Microbiology Maharishi Markandeshwar Institute of Medical Sciences, MMU, Mullana, Ambala Haryana, India
${ }^{7}$ Senior Resident, Department of Microbiology Maulana Azad Medical College and Lok Nayak Hospital, New Delhi, India
}

DOI: $10.36348 /$ sjm.2020.v05i05.004 $\quad$ | Received: 30.04 .2020 | Accepted: 08.05.2020 | Published: 17.05 .2020

*Corresponding author: Dr. Pragyan Swagatika Panda

\section{Abstract}

Background: Escherichia coli is one of the commonest pathogens causing community acquired Urinary Tract Infection (UTI). Increase in resistance to antibiotics and limited option of oral antibiotics for UTI calls for search for better alternative options. Extended Spectrum Beta Lactamase producing E. coli (ESBL- EC) further reduces the therapeutic options complicating the management. Nitrofurantoin (NIT) is one of the commonly prescribed oral drugs and Fosfomycin (FO) though an old drug is regaining its medical importance. The aim of the study was to study and compare the sensitivity of NIT and FO in ESBL-EC causing UTI. Material and Method: A total of 215 mid-stream urine samples showing growth of $E$. coli were collected and ESBL-EC identified. Antibiotic sensitivity testing was done by Kirby Bauer disc diffusion method as per CLSI 2019 guidelines using NIT $(300 \mu \mathrm{g})$ and FO $(200 \mu \mathrm{g})$ discs. The results were compared. Result: Out of 215 urine sample, 57(26.5\%) isolates showed resistance to NIT while FO resistance was observed only in $11(5.1 \%)$ of the isolates. Amongst isolated E. coli, $122(56.7 \%)$ were extended spectrum beta lactamase (ESBL). Among the resistant isolates, ESBL producers were 45.5\% (5 out of 11) and 57.8\% (33 out of 57) for FO and NIT respectively. Conclusion: Increase in resistance trend in NIT calls for search of other oral options for treatment and FO promises to be one of them.

Keywords: Nitrofurantoin, Fosfomycin, Escherichia coli, ESBL.

Copyright @ 2020: This is an open-access article distributed under the terms of the Creative Commons Attribution license which permits unrestricted use, distribution, and reproduction in any medium for non-commercial use (NonCommercial, or CC-BY-NC) provided the original author and source are credited.

\section{INTRODUCTION}

Urinary tract infections (UTI) are amongst the commonly treated infections in primary health care facilities. In both community and hospital settings, UTIs account for most common bacterial infections in children <2years of age [1]. It has been found that at least $40 \%$ of women develop UTI minimum once in their lifetime and adult women are 30 times more prone to develop UTI than males [2]. In cases of congenital anomalies of urinary tract, UTI can lead to renal scarring in infants who in adulthood may lead to complications like hypertension, proteinuria, renal damage or even renal failure [3].

Availability of over the counter medications and antibiotic misuse has led to surge of antibiotic resistant uropathogens. It has led to increased morbidity; longer hospital stays and increased treatment expenditure. Production of Extended Spectrum Beta Lactamase (ESBL) by E.Coli confers resistance to penicillins, cephalosporins, monobactam and ESBL producers show co- resistance to other commonly used antimicrobials like fluoroquinolones, co-trimoxazole, and aminoglycosides. Currently this further reduces the therapeutic options complicating the management of UTI. However, it has been observed that many of the resistant organisms still retain their susceptibility to two of the older oral drugs Nitrofurantoin (NIT) and Fosfomycin (FO) [4]. CLSI 2019 had limited FO testing as a first line drug only for uropathogenic E. coli amongst Enterobacteriaceae. Both NIT and FO share common properties like higher concentrations in urinary tracts, minimal impact on gastrointestinal flora and lower chances of acquiring resistance. Hence study of sensitivity of ESBL E. coli (ESBL-EC) to NIT and 
FO is important to provide clinician an additional option for treating UTI with oral antimicrobial agent. So, this study was aimed at assessing and comparing the sensitivity of UTI causing ESBL-EC to NIT and FO.

\section{MATERIAL AND METHODS}

All the subjects of $>18$ years and of either sex with clinical suspicion of UTI attending OPD/IPD of a tertiary care Super specialty Hospital in New Delhi were enrolled for this prospective study carried out over a period of 6 months (July-December 2019). Demographic details like name, age, sex, OPD/IPD were noted. Clean catch midstream urine samples of the study subjects were plated on CLED Agar (Himedia, Mumbai, India) and were incubated overnight at $37^{\circ} \mathrm{C}$. The culture plates were read next morning and growth of Escherichia coli was identified as per standard microbiological techniques and was subjected to routine antibiotic testing using Kirby Bauer Disk Diffusion Method according to CLSI 2019 guidelines. As per the CLSI guidelines, ESBL producers were identified using
Ceftazidime $(30 \mu \mathrm{g})$ and ceftazidime-clavulanic acid $(30 \mu \mathrm{g} / 10 \mu \mathrm{g})$. Among the isolates, sensitivities for Nitrofurantoin $(300 \mu \mathrm{g})$ and fosfomycin $(200 \mu \mathrm{g})$ were observed. Data were expressed in terms of percentage.

\section{RESULTS}

A total of 215 [117 (54.4\%) female and 98 (45.6\%) male] culture positive urine samples showing growth of Escherichia coli were included in this study. Out of the total isolates tested for NIT sensitivity, $57(26.5 \%)$ were resistant, $4(1.8 \%)$ were intermediate sensitive and $154(71.6 \%)$ of isolates were sensitive. In contrast, majority of the isolates [204 (94.9\%)] were sensitive to FO and only $11(5.1 \%)$ were resistant to it. Amongst isolated E. coli, $122(56.7 \%)$ were ESBL producers while $93(43.3 \%)$ were non-ESBL producers. Among the resistant isolates, ESBL producers were $45.5 \%$ (5 out of 11) and $57.8 \%$ (33 out of 57) for FO and NIT respectively (Table 1 ). The figures were $54.5 \%$ (6 out of 11) and 42.2\% (24 out of 57) for FO and NIT respectively among ESBL non producers.

Table-1: Comparison of NIT and FO resistance in ESBL positive and Negative isolates

\begin{tabular}{|l|l|l|}
\hline & ESBL PRODUCER & ESBL NON-PRODUCER \\
\hline FO- ${ }^{*}(\mathrm{~N}=11)$ & $5(45.5 \%)$ & $6(54.5 \%)$ \\
\hline NIT-R ${ }^{*}(\mathrm{~N}=57)$ & $33(57.8 \%)$ & $24(42.2 \%)$ \\
\hline \multicolumn{2}{|c|}{$* \mathrm{R}=$ Resistant }
\end{tabular}

\section{DISCUSSION}

With the increasing antimicrobial resistance, it has become very important to know the local resistance trends of the commonly isolated organisms to the available treating options. Currently a rising trend of ESBL uropathogens is observed both within health care settings and in the community. Since UTI treatment begins with empirical antimicrobial agents, the need for a high efficacy antimicrobial agent for clinical and microbiological success cannot be overemphasized. So it is imperative to search for oral therapeutic options having higher therapeutic profile with minimum side effects for ESBL-EC. Fosfomycin is a novel antibiotic which shows good efficacy against UTI causing E.coli and NIT is still widely used agent for the treatment of UTI. Earlier FO was used for treating UTI caused by Enterobacteriaceae. So, majority of the previous studies pertaining to FO were focused on common uropathogens. But CLSI 2019 decreased treatment horizon of FO to only E. coli and Enterococcus spp. causing UTI. Hence study of sensitivity pattern of uropathogenic ESBL-EC to FO is scarce. This prompted us to do the current study in which we tried to assess sensitivity of ESBL-EC to FO and NIT so as to guide the clinician regarding an optimal empirical therapy of these infections.

This study included 215 urinary E. coli isolates, majority $(54.5 \%)$ were from female patients. Females have a high propensity for UTI as compared to male due to certain anatomical difference in lower urinary tract. In our study $71.6 \%$ of uropathogenic E.coli were sensitive to NIT. In their study, Raja, et al. reported sensitivity to NIT as $93 \%$ amongst the E. coli isolates[5]. Various other studies have reported NIT sensitivity to be around $80 \% \%$ to $87 \%$ respectively [6, 7]. In India, sensitivity rate among $E$. coli in NIT range from 76 to $95 \%$ with higher resistance rate seen in inpatients [8]. This variation in sensitivity pattern may be attributed to current inappropriate use and over the counter availability of this drug in different geographical locations in our country.

In our study $94.9 \%$ of the isolates were found to be sensitive to FO. Our finding is similar to that observed by Banerjee, et al. wherein $95.18 \%$ isolates were susceptible to FO [9]. A study in Turkey in 2019 reported sensitivity rates of E.coli for NIT and FO to be $94 \%$ and $98 \%$ respectively [10]. Long term clinical studies have shown so far that only around 3\% UTI causing bacteria have developed resistance to Fosfomycin [11]. But, our study reports slightly higher resistance $(5.1 \%)$. This difference can be explained in terms of widespread use of Fosfomycin since it is an alternative oral antimicrobial option to Nitrofurantoin.

Our study showed that $122(56.7 \%)$ of the uropathogenic E.coli were ESBL producers of which $45.5 \%$ (5 out of 11 ) were resistant to FO and $57.8 \%$ (33 out of 57)were resistant to NIT. In a study by Gupta, et al. amongst 150 uropathogenic E.coli, $52.6 \%$ of isolates were ESBL producers, and all of them were 
susceptible to FO [12]. In their study Mittal et al. showed $100 \%$ of isolated uropathogenic E. coli strains to be sensitive to FO [13]. Another study from Brazil reported $98.8 \%$ sensitivity rate for fosfomycin in $E$. coli from community acquired UTIs despite the heavy usage of this antibiotic [13]. Fosfomycin resistance in the clinical isolates is rare because of higher concentration of fosfomycin in the urinary tract. Our findings of $>95 \%$ fosfomycin susceptibility among ESBL producing Enterobacteriacae supports the view of use of this antibiotic in uncomplicated UTIs as reported before [5].

Both NIT and Fosfomycin are good oral antimicrobial agents which are highly effective in treating UTI and have low resistance rates. Although NIT has been first line drug for UTI from a long time, FO was a reserve drug. It has been suggested that since the efficacy of single dose of Fosfomycin is comparable to 7 days treatment with NIT, Fosfomycin can have better patient compliance [14]. Hence it can be considered as a good alternative option to NIT for treating ESBL-EC UTI. In CLSI 2019 both NIT and FO have been listed as first line antibiotics for treating uncomplicated UTI. This study thus compares sensitivity pattern of these first line drugs. Our study showed an increasing trend in resistance to one of the widely prescribed oral antibiotics, NIT which warrants us to search for alternatives to it. Fosfomycin is an old drug gaining importance because of the low propensity for resistance and also it is available in oral form which leads to better patient compliance. Hence, our study results can help in providing the clinicians an alternative option to NIT in treating ESBL-EC UTI.

\section{CONCLUSION}

Increasing resistance to Nitrofurantoin by uropathogenic ESBL E.Coli is a matter of concern currently. Because of the low propensity for resistance fosfomycin is a good oral alternative for NIT. But its rampant and uncontrolled usage should be minimized by adhering to proper antibiotic policies.

\section{REFERENCES}

1. Hanna-Wakim, R. H., Ghanem, S. T., El Helou, M. W., Khafaja, S. A., Shaker, R. A., Hassan, S. A., ... \& Bakhash, M. (2015). Epidemiology and characteristics of urinary tract infections in children and adolescents. Frontiers in cellular and infection microbiology, 5,45 .

2. Foxman, B. (2002). Epidemiology of urinary tract infections: incidence, morbidity, and economic costs. The American journal of medicine, 113(1), 513 .

3. Tan, C. W., \& Chlebicki, M. P. (2016). Urinary tract infections in adults. Singapore medical journal, 57(9), 485.
4. Gardiner, B. J., Stewardson, A. J., Abbott, I. J., \& Peleg, A. Y. (2019). Nitrofurantoin and fosfomycin for resistant urinary tract infections: old drugs for emerging problems. Australian prescriber, 42(1), 14.

5. Auer, S., Wojna, A., \& Hell, M. (2010). Oral treatment options for ambulatory patients with urinary tract infections caused by extendedspectrum- $\beta$-lactamase-producing Escherichia coli. Antimicrobial agents and chemotherapy, 54(9), 4006-4008.

6. Singhal, A., Sharma, R., Jain, M., \& Vyas, L. (2014). Hospital and community isolates of uropathogens and their antibiotic sensitivity pattern from a tertiary care hospital in North West India. Annals of medical and health sciences research, 4(1), 51-56.

7. Prasada, S., Bhat, A., Bhat, S., Mulki, S. S., \& Tulasidas, S. (2019). Changing antibiotic susceptibility pattern in uropathogenic Escherichia coli over a period of 5 years in a tertiary care center. Infection and Drug Resistance, 12, 1439.

8. Shakti, L., Veeraraghvan, B.(2015). Advantages and limitations of nnitrofurantoin in multi-drug resistant indian scenario. Ind J Med Microbiol, 33(4):477-81.

9. Banerjee, S., Sengupta, M., \& Sarker, T. K. (2017). Fosfomycin susceptibility among multidrugresistant, extended-spectrum beta-lactamaseproducing, carbapenem-resistant uropathogens. Indian journal of urology: IJU: journal of the Urological Society of India, 33(2), 149.

10. Coşkun, U. S. Ş. (2019). Fosfomycin and nitrofurantoin susceptibilities of Escherichia coli and Klebsiella pneumoniae isolates producing extanded spectrum Beta-lactamase causing urinary tract infections. Çăgdaş Tıp Dergisi, 9(1), 55-58.

11. Arca, P., Reguera, G., \& Hardisson, C. (1997). Plasmid-encoded fosfomycin resistance in bacteria isolated from the urinary tract in a multicentre survey. The Journal of antimicrobial chemotherapy, 40(3), 393-399.

12. Gupta, V., Rani, H., Singla, N., Kaistha, N., \& Chander, J. (2013). Determination of extendedspectrum $\beta$-lactamases and AmpC production in uropathogenic isolates of Escherichia coli and susceptibility to fosfomycin. Journal of laboratory physicians, 5(2), 90.

13. Mittal, S., Sharma, M., \& Chaudhary, U. (2015). Fosfomycin use in multi drug resistant uropathogenic Escherichia coli. Infectious Disorders-Drug Targets (Formerly Current Drug Targets-Infectious Disorders), 15(3), 196-201.

14. Parate, S. (2016). A comparative study of Fosfomycin and NIT in acute uncomplicated UTI. Int J of Med research and preview, 4(5):787-91. 